Article

\title{
Functional Characterization of the Steroid Reductase Genes GmDET2a and GmDET2b from Glycine max
}

\author{
Weige Huo ${ }^{1,+}$, Bodi Li ${ }^{1,2,+}$, Jiebing Kuang ${ }^{1,2}$, Pingan $\mathrm{He}^{1,2}$, Zhihao $\mathrm{Xu}^{1,2}$ \\ and Jinxiang Wang ${ }^{1,2, *}$ \\ 1 State Key Laboratory for Conservation and Utilization of Subtropical Agro-bioresources, \\ South China Agricultural University, Guangzhou 510642,China; wghuo@cau.edu.cn (W.H.); \\ 011068ch@stu.scau.edu.cn (B.L.); binel168@163.com (J.K.); pinganhe@stu.scau.edu.cn (P.H.); \\ xuzhihao@stu.scau.edu.cn (Z.X.) \\ 2 Root Biology Center, College of Natural Resources and Environment, South China Agricultural University, \\ Guangzhou 510642, China \\ * Correspondence: jinxwang@scau.edu.cn; Tel.: +86-020-8528-0156 \\ + These authors contributed equally to this work.
}

Received: 30 January 2018; Accepted: 28 February 2018; Published: 3 March 2018

\begin{abstract}
Brassinosteroids are important phytohormones for plant growth and development. In soybean (Glycine max), BR receptors have been identified, but the genes encoding BR biosynthesis-related enzymes remain poorly understood. Here, we found that the soybean genome encodes eight steroid reductases (GmDET2a to GmDET2h). Phylogenetic analysis grouped 105 steroid reductases from moss, fern and higher plants into five subgroups and indicated that the steroid reductase family has experienced purifying selection. GmDET2a and GmDET2b, homologs of the Arabidopsis thaliana steroid $5 \alpha$-reductase AtDET2, are proteins of 263 amino acids. Ectopic expression of GmDET2a and GmDET2b rescued the defects of the Atdet2-1 mutant in both darkness and light. Compared to the mutant, the hypocotyl length and plant height of the transgenic lines GmDET2a and $G m D E T 2 b$ increased significantly, in both darkness and light, and the transcript levels of the BR biosynthesis-related genes CPD, DWF4, BR6ox-1 and BR6ox-2 were downregulated in GmDET2aOX-23 and GmDET2bOX-16 lines compared to that in Atdet2-1. Quantitative real-time PCR revealed that GmDET2 $a$ and GmDET2b are ubiquitously expressed in all tested soybean organs, including roots, leaves and hypocotyls. Moreover, epibrassinosteroid negatively regulated GmDET2a and GmDET2b expression. Sulfate deficiency downregulated GmDET2a in leaves and GmDET2b in leaves and roots; by contrast, phosphate deficiency upregulated $G m D E T 2 b$ in roots and leaves. Taken together, our results revealed that GmDET2a and GmDET2b function as steroid reductases.
\end{abstract}

Keywords: GmDET2; steroid reductase; brassinosteroid; soybean

\section{Introduction}

Brassinosteroids (BRs), as classical phytohormones, play important roles in many aspects of plant growth and development including root growth and development [1,2], stomatal development [3], seed germination [4], skotomorphogenesis [5,6], phototropism [7], nodulation [8], immunity responses [9] and abiotic stress responses [10,11].

In animals, steroid hormones are perceived through nuclear receptors. By contrast, in plants, BR is sensed by the BR receptors such as brassinosteroid insensitive 1 (BRI1) in the plasma membranes [12,13]. Recently, the linear BR signaling pathway in Arabidopsis has been revealed. In the absence of BRs, BRI1 is bound by the membrane-located kinase BKI1 [14]. Upon reception of BR, BRI1 disassociates BKI1 [14] and physically interacts with the membrane kinase BAK1, which is considered as a co-receptor [15]. BRI1 and BAK1 transphosphorylate each other [14]. Brassinosteroid insensitive 2 
(BIN2) functions downstream of BRI1. As a highly conserved GSK kinase and negative regulator of BR signaling, BIN2 phosphorylates transcription factors brassinazole-resistant 1 (BZR1) and BZR2 and in turn inactivated them [16-19]. Conversely, protein phosphatase 2A (PP2A) mediates the dephosphorylation of BZR1 to activate it [20]. When BR levels are high, BR signaling leads to the inactivation of BIN2. Accordingly, the dephosphorylated BZR1 and BZR2 shuttle between the cytoplasm and nucleus, binding promoters of several downstream genes, further strengthening BR signaling [21]. BIN2 and BZR1 have been reported to be regulated by the proteasome-dependent pathway in Arabidopsis [18,22].

Previous studies have revealed that Arabidopsis has three functional BR receptors, namely BRI1, BRL1 and BRL3, while AtBRL2 appears to be nonfunctional in BR signaling [23,24]. The rice genome encodes four BR receptors, OsBRI1, OsBRL1, OsBRL2 and OsBRL3 [25,26]. BR receptors have also been identified in tomato [27], pea [28], barley [29], cotton [30] and soybean [31,32].

As reported, more than $50 \mathrm{BRs}$ have been isolated and identified from several plant species [33]. Among them, brassinolide (BL) is the most active BR. Based on previous studies, the first reaction of BL biosynthesis is the formation of campestanol (CN) from campesterol (CR); the second step is the conversion of $\mathrm{CN}$ to castasterone (CS) via $\mathrm{C} 6$ oxidation; and the final step is the conversion of $\mathrm{CS}$ to BL [34]. The steroid $5 \alpha$-reductase, DET2, which hydrogenates a (24R)-24-methylcholest-4-en-3-one intermediate to convert $\mathrm{CR}$ into $\mathrm{CN}$, catalyzes the rate-limiting step in BR biosynthesis [35,36]. The Atdet2-1 mutant has a dwarf phenotype; its growth and development in the dark and light both retarded significantly [5].

In 1990, a Japanese group reported that BR promotes adventitious rooting in soybean hypocotyl cuttings [37]. Later, BR was found to promote stem growth in soybean [38], and the application of $B R$ increased soybean tolerance to drought and increased the concentration of soluble sugar and proline [39]. Additionally, BR increases the expression of soybean small auxin up RNA 6B (SAU 6B) in elongating epicotyls, in a time-dependent manner [38]. These studies indicate that BRs regulate soybean growth, development and stress responses at physiological and molecular levels, but the underlying mechanisms are poorly understood.

Although the BR receptors in soybean have been identified [31,32], BR biosynthesis remains elusive at the molecular level. In this study, we identified eight steroid reductase genes (GmDET2a to GmDET2h) in soybean. Phylogenetic analysis indicated that a total of 105 steroid reductase (dehydrogenases) from moss, fern and higher plants can be grouped into five subgroups, and steroid reductase is subjected to purifying selection during evolution. GmDET2a and GmDET2b were negatively regulated by epibrassinosteroid. Phosphate (Pi) starvation induced the expression of $G m D E T 2 b$ in leaves and roots, and sulfate deficiency downregulated the abundance of GmDET2b in leaves and roots. Ectopic expression of GmDET2a and GmDET2b rescued the defects of det2-1 mutants both in darkness and light and altered the transcript levels of BR biosynthesis-related genes.

\section{Results}

\subsection{Identification of Steroid Reductases in Soybean}

To identify steroid reductases in the soybean proteome, we took two approaches. First, we searched the soybean proteome for the PF02544 domain (http:/ / pfam.xfam.org/), the signature domain of steroid reductase [40], and found a total of six putative steroid reductase. Second, the amino acid sequence of the known steroid reductase AtDET2 was used to search the soybean proteome with BLAST-P (http: / /blast.ncbi.nlm.nih.gov), and the two proteins encoded by Glyma.07g144400 and Glyma.11g010000 showed the highest identity and similarity to AtDET2. Collectively, the soybean genome encodes eight steroid reductase (Table 1). Although the PF02544 domain was not found in Glyma.07g144400 and Glyma11g010000, the BLAST search against the soybean proteome with Arabidopsis DET2 showed that Glyma.07g144400 and Glyma.11g010000 appear to have a similar function to that of AtDET2, and thus, they have been named GmDET2a and GmDET2b, respectively. 
Table 1. General information about the steroid reductases in soybean based on bioinformatics analysis.

\begin{tabular}{ccccccc}
\hline Name & Locus & Alias & EST & Length (AA) & Putative Localization & Int/Ext \\
\hline GmDET2a & Glyma.07G144400 & Glyma07g17410 & yes & 263 & Plas & $0 / 1$ \\
GmDET2b & Glyma.11G010000 & Glyma11g01210 & yes & 263 & Plas & $0 / 1$ \\
GmDET2c & Glyma.02G081200 & Glyma02g08970 & yes & 266 & Cyto & $1 / 2$ \\
GmDET2d & Glyma.02G081100 & Glyma02g08950 & yes & 266 & Plas & $1 / 2$ \\
GmDET2e & Glyma.11G110300 & Glyma11g11780 & yes & 342 & Nucl & $5 / 6$ \\
GmDET2f & Glyma.14G043500 & Glyma14g04780 & yes & 309 & Plas & $3 / 4$ \\
GmDET2g & Glyma.02g273300 & Glyma02g44090 & yes & 309 & $?$ & $4 / 5$ \\
GmDET2h & Glyma.19g261600 & Glyma19g45120 & yes & 317 & $?$ & $2 / 3$ \\
\hline
\end{tabular}

Putative cell localization of soybean steroid reductases was predicted with PSORT. Based on the released genome sequences and cDNA sequences of soybean, the number of introns and exons were determined through GSDS as described in the Materials and Methods. EST, expressed sequence tag; Plas, plasma membrane; Nucl, nucleus; Cyto, cytoplasm; AA, amino acid; Int, intron; Ext, extron.

In order of BLAST E-value, six proteins, in addition to Glyma.07g144400 and Glyma.11g010000, were found. In order, they are Glyma.02G081200, Glyma.02G081100, Glyma.11G110300, Glyma.14G043500, Glyma.02g273300 and Glyma.19g261600. The six related genes have been named GmDET2c, GmDET2d, GmDET2e, GmDET2f, GmDET2g and GmDET2h, respectively, as listed in Table 1. GmDET2c, GmDET2d and GmDET2 $g$ are located on chromosome 2, GmDET2b and GmDET2e on chromosome 11, GmDET2a on chromosome 7, GmDET2f on chromosome 12 and GmDET2h on chromosome 19. The amino acid length of steroid reductases in soybean ranged between 263 and 342 amino acids.

Based on the data in Phytozome (www.phytozome.org), the gene structure of soybean DETs was analyzed with the GSDS2.0 program (http://60.209.29.212/). The soybean DET2 family can be grouped into three subgroups (intro-less, one intron and two or more introns); both GmDET2a and $G m D E T 2 b$ are intron-less genes; $G m D E T 2 c$ and GmDET2d have one intron; while GmDET2e, GmDET2f, GmDET2 $g$ and GmDET2h contain 3, 5, 4 and 2 introns, respectively (Figure 1A).

Based on previous RNA-Seq study on soybean [41], we found that GmDET2a and GmDET2b are ubiquitously expressed in young leaves, flowers, pods, seeds, roots and nodules, with relatively higher transcript abundance in roots and nodules (Figure 1). The transcript levels of GmDET2d and GmDET2h are higher in roots than in other organs, while those of GmDET2b and GmDET2d are higher in nodules. The transcripts of GmDET2c and GmDET2h are higher in leaves, and the expression levels of GmDET2c and $G m D E T 2 h$ are higher in flowers. Interestingly the transcripts of all eight steroid reductase genes can be detected in seeds, with transcripts of GmDET2c in 10 days after fertilization (DAF) seeds being significantly higher. The abundance of GmDET2c and GmDET2h is somewhat higher in one-cm pod, pod shell 10 DAF and pod shell 14 DAF (Figure 1B). 


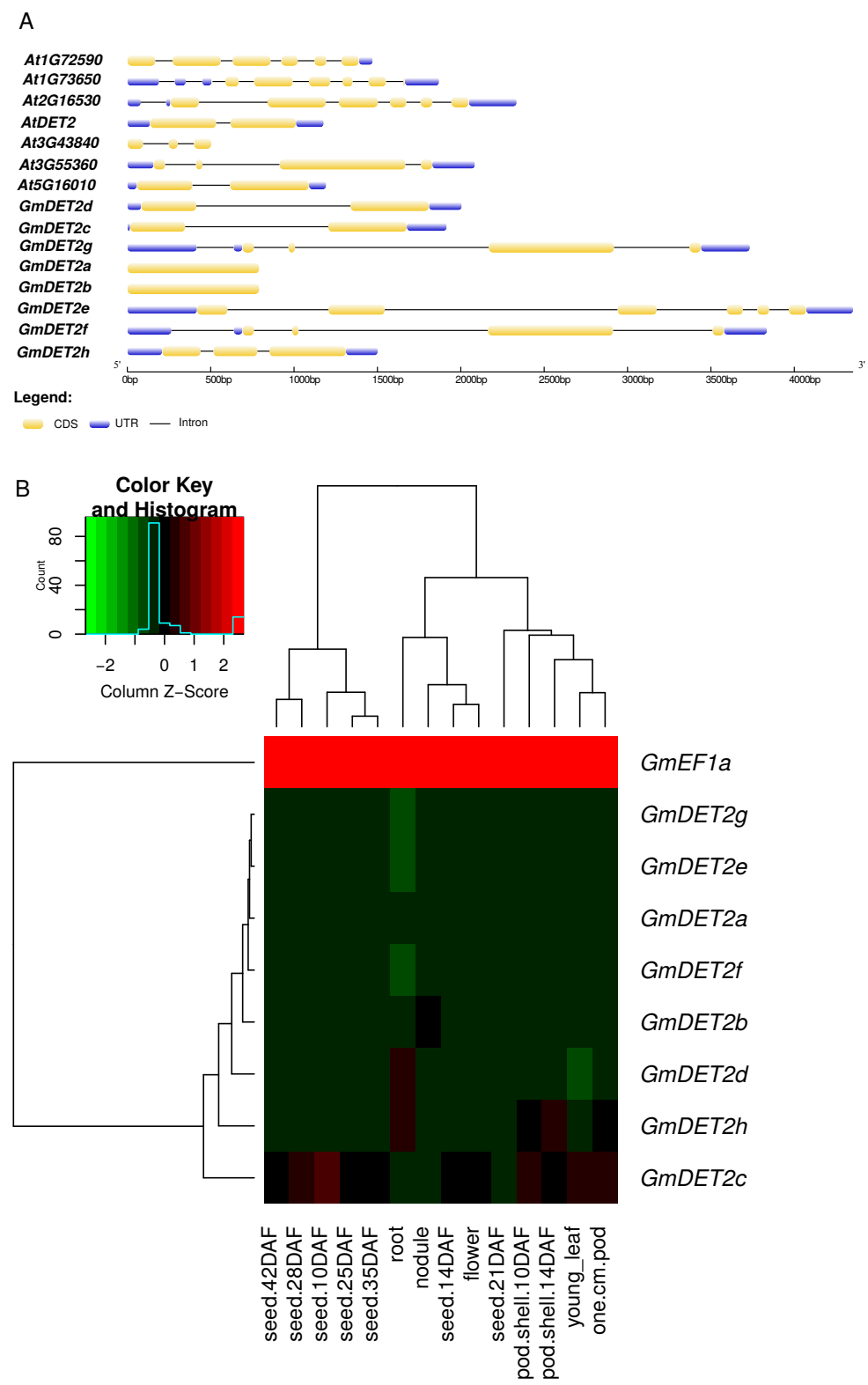

Figure 1. Gene structure of Arabidopsis and soybean steroid reductase and transcript levels of the soybean steroid reductase family. The gene structure of seven and eight steroid reductase from Arabidopsis and soybean are shown (A). CSDS2.0 was used to analyze the CDS (coding DNA sequence), UTR (untranslated region) and intron of these genes. The heat map (B) demonstrates the expression levels of GmDET2a to GmDET2h. The normalized expression data from RNA-Seq were downloaded from SoyBase [41]. DAF, day after fertilization.

\subsection{Phylogenetic Analysis of Steroid Reductases}

We aligned the amino acid sequences of eight soybean DET2s with 12 homologs from the reference species Arabidopsis thaliana and Oryza sativa and reconstructed the phylogenetic tree of DET2s with MEGA6 [42]. As expected, GmDET2a and GmDET2b grouped together with AtDET2 and Os01g63260. GmDET2c and GmDET2d clustered with At5g16010, GmDET2e with Os04g48750, GmDET2f and GmDET2g with At3G55360 and GmDET2h with At1g73650. In short, DET2s from soybean, Arabidopsis and rice can be grouped into five sub-clades (Figure 2).

To extensively decipher the evolutionary history of steroid reductase in the kingdom Plantae, we further collected steroid reductases from multiple plants, including Physcomitrella patens, 
Selaginella moellendorffii, Oryza sativa, Brachypodium distachyon, Sorghum bicolor, etc. After alignment with the CLUSTALW program, sequences were analyzed with MrBayes 3.2 [43]. As shown in Figure 3, steroid reductases of Plantae are grouped into five subgroups. Of note, except for group V, all subgroups have members from Physcomitrella patens and Selaginella moellendorffii. This indicates that higher plants have retained ancestral steroid reductase genes and that steroid reductase plays an important role in growth and development.

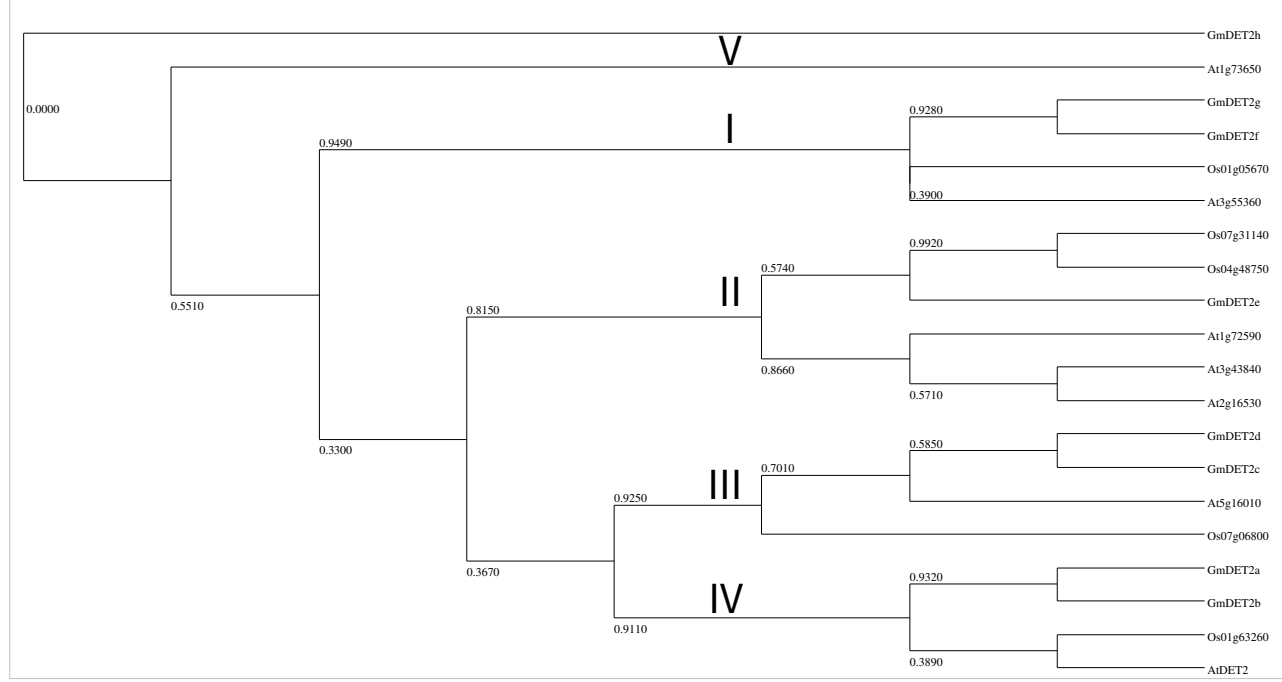

Figure 2. Molecular Phylogenetic analysis by the maximum likelihood method. The evolutionary history was inferred by using the maximum likelihood method based on Jones et al.'s w/freq. model [44]. The tree with the highest log likelihood (-2073.6839) is shown. The percentage of trees in which the associated taxa clustered together is shown next to the branches. Initial tree(s) for the heuristic search were obtained automatically by applying the neighbor-join and BioNJalgorithms to a matrix of pairwise distances estimated using a JTTmodel and then selecting the topology with the superior log likelihood value. A discrete gamma distribution was used to model evolutionary rate differences among sites (five categories $(+G$, parameter $=5.1876)$ ). The tree is drawn to scale, with branch lengths measured in the number of substitutions per site. The analysis involved 20 amino acid sequences. All positions containing gaps and missing data were eliminated. There was a total of 74 positions in the final dataset. Evolutionary analyses were conducted in MEGA6 [42]. Steroid reductases from soybean, Arabidopsis and rice can be grouped into five subgroups (I, II, III, IV and V).

To identify the selection pressure during evolution of plant steroid reductases, we explored the ratios of $\mathrm{Ka}$ to $\mathrm{Ks}(\mathrm{Ka} / \mathrm{Ks})$ of steroid reductase from P. patens, S. moellendorffii, O. sativa, B. distachyon, G. max and A. thaliana. Generally, genes are subjected to three kinds of selection, namely positive selection $(\mathrm{Ka} / \mathrm{Ks}>1)$, neutral selection $(\mathrm{Ka} / \mathrm{Ks}=1)$, and negative or purifying selection $(\mathrm{Ka} / \mathrm{Ks}<1)$. Results in Figure 4 showed that plant steroid reductase are subjected to purifying selection, except for three branches marked in red, in which the Ka/Ks are greater than one (Figure 4). 


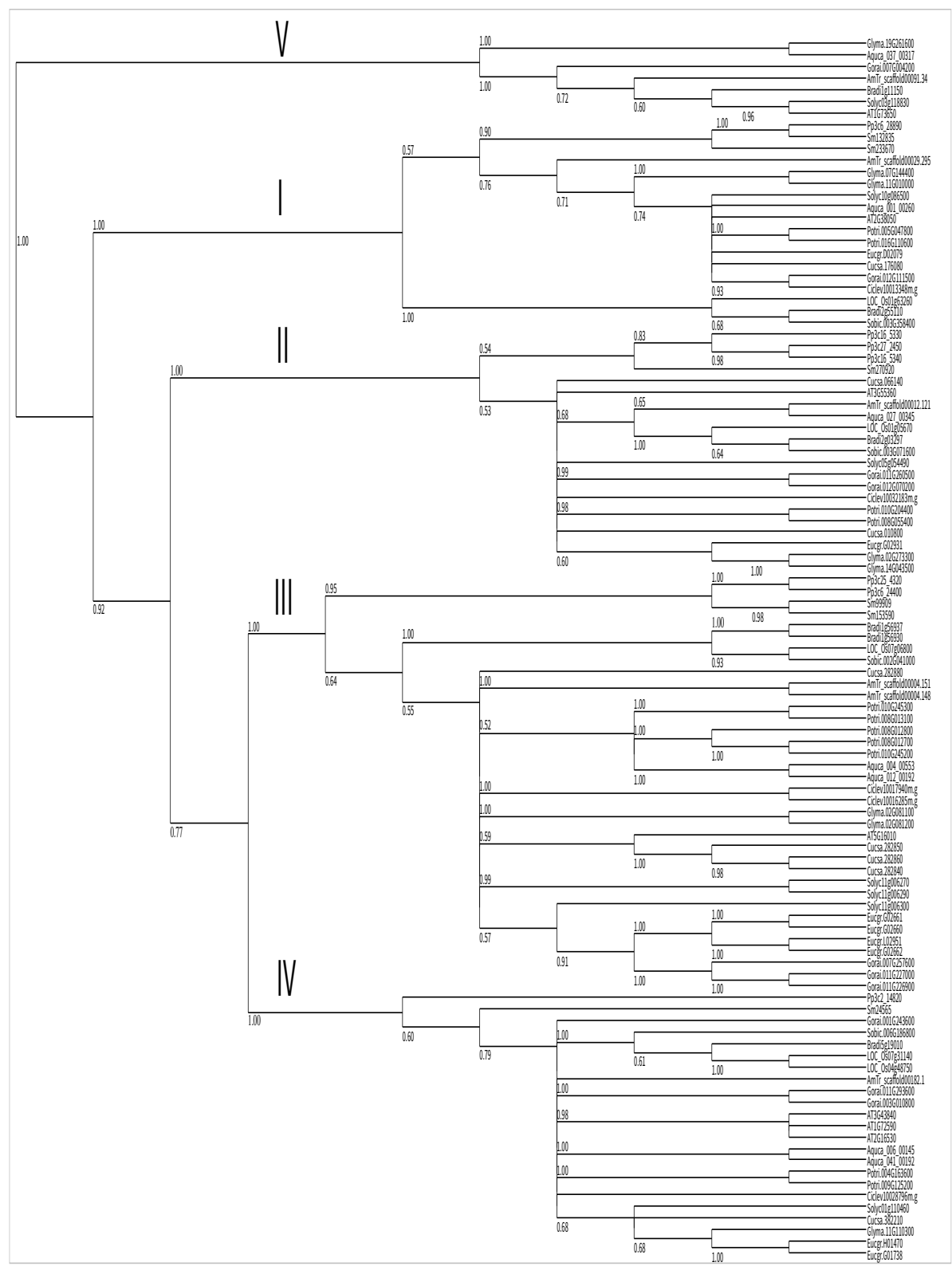

Figure 3. Molecular phylogenetic analysis of steroid reductases in planta. BLASTP was used to search for BR receptor homologues in different plant species. A total of 105 steroid reductase as shown in Additional File 1 (Spreadsheet S1) was analyzed from Physcomitrella patens (Pp), Selaginella moellendorffii (Sm), Cucumis sativus (Cucsa), Populus trichocarpa (Potri), Eucalyptus grandis (Eucgr), Citrus clementina (Cicle), Solanum lycopersicum (Solyc), Glycine max (Glyma), Arabidopsis thaliana (AT), Brachypodium distachyon (Bradi), Oryza sativa (Os), Amborella trichopoda (AmTr), Gossypium raimondii (Gorai), Aquilegia coerulea (Aqcoe) and Sorghum bicolor (Sobic). Values expressed below the branches are the probability of the bootstrap value with 1000 repeats. MrBayes 3.2 [43] software was used to reconstruct the phylogenetic tree, as described in the Methods Section. 


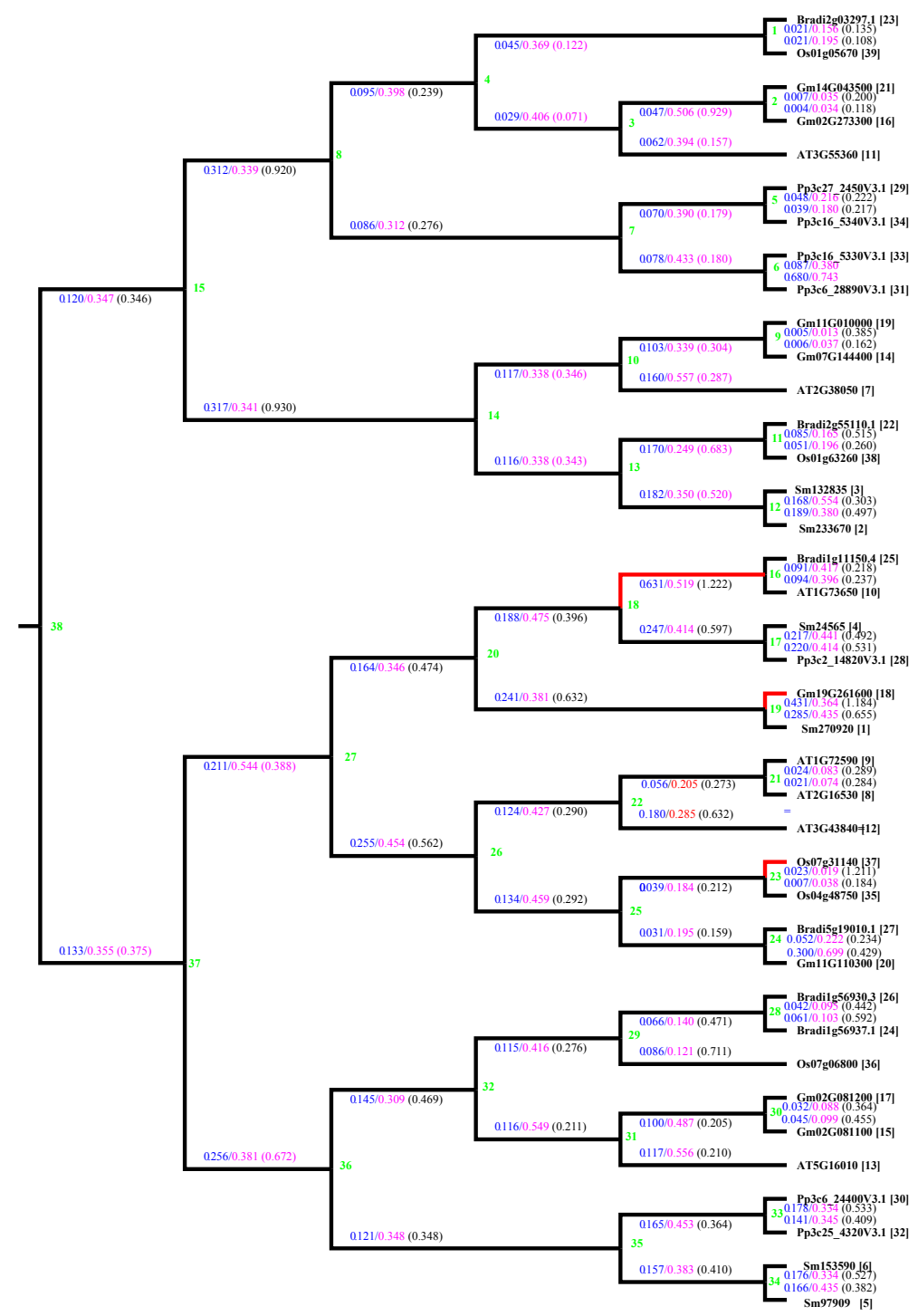

Figure 4. Estimation of $\mathrm{Ka} / \mathrm{Ks}$ in steroid reductase genes from soybean, common bean, Arabidopsis, rice. The cDNA sequences and amino acid sequences of steroid reductase from moss Physcomitrella patens (Pp), fern Selaginella moellendorffii (Sm), Glycine max (Gm), Arabidopsis thaliana (AT), Brachypodium distachyon (Bradi) and Oryza sativa (Os) were used to estimate Ka/Ks (http:/ / services.cbu.uib.no/tools/kaks). The Ka and Ks values in each node and branch are marked in blue and red, respectively. A total of 38 nodes is shown in green, and the ratios of $\mathrm{Ka} / \mathrm{Ks}$ in each branch are indicated in parentheses. The ratios of $\mathrm{Ka} / \mathrm{Ks}$ in most branches are less than one, except for three branches in red.

\subsection{Transcript Levels of GmDET2a and GmDET2b in the Seedling Stage}

Taking into consideration the important roles of brassinosteroid in plant growth and development, that GmDET2 appear to be involved in BR biosynthesis, and that GmDET2a and GmDET2b are the closest homologs of AtDET2, we thus focused on them in subsequent experiments. We first employed quantitative real-time PCR (qRT-PCR) to detect the transcripts of GmDET2a and GmDET2b in the seedlings of soybean. As shown in Figure 5, qRT-PCR results showed that GmDET2a and GmDET2b were both expressed in apical buds, leaves, hypocotyls, primary root (PR) tips and lateral roots. The transcripts of GmDET2 $a$ in apical buds, PR tips and lateral roots are somewhat higher than those in leaves and hypocotyls (Figure 5). A similar pattern was observed in the case of GmDET2b (Figure 5). 


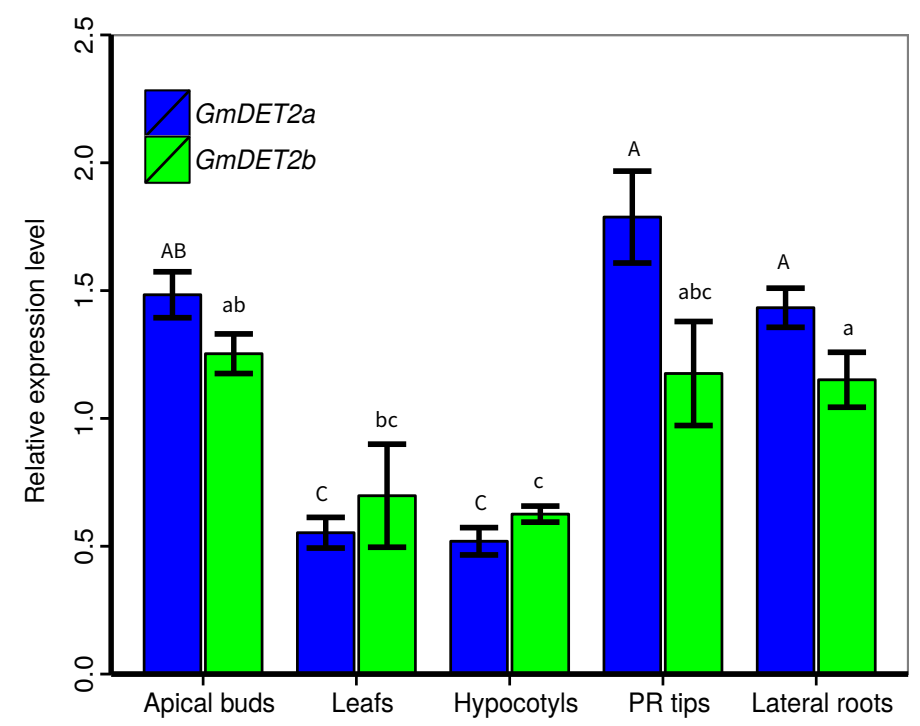

Figure 5. Transcript levels of GmDET2a and GmDET2b in soybean seedlings. RNA was extracted from seven-day-old soybean YC03-3. Quantitative real-time PCR was exploited to detect the transcripts of GmDET2a (in blue) and GmDET2b (in green) in apical buds, leaves, hypocotyls, primary root (PR) tips and lateral roots. Results are the means \pm SD from four replicates. One-way ANOVA was used to compare the differences between organs, with uppercase letters or lowercase letters indicating the difference at the 0.05 level.

\subsection{GmDET2a and GmDET2b Were Negatively Regulated by Epibrassinosteroid}

As previously reported, AtDET2 is negatively regulated by exogenous BR [45,46]. To explore this relationship in soybean, we looked at the expression pattern of GmDET2a and GmDET2b in soybean seedlings treated with epibrassinosteroid (EBL) for $6 \mathrm{~h}$. As shown in Figure 6A, in general, GmDET2a was negatively regulated by $6 \mathrm{~h}$ EBL treatment. The abundance of GmDET2a was significantly reduced in apical buds $(p<0.01)$, leaves $(p<0.01)$, primary root (PR) tips $(p<0.01)$ and lateral roots $(p<0.05)$, but not negatively regulated by EBL in hypocotyls. GmDET2b was negatively regulated by EBL in only primary root tips $(p<0.01)$ and lateral roots $(p<0.05)$, but not in apical buds, leaves and hypocotyls (Figure 6B).

A

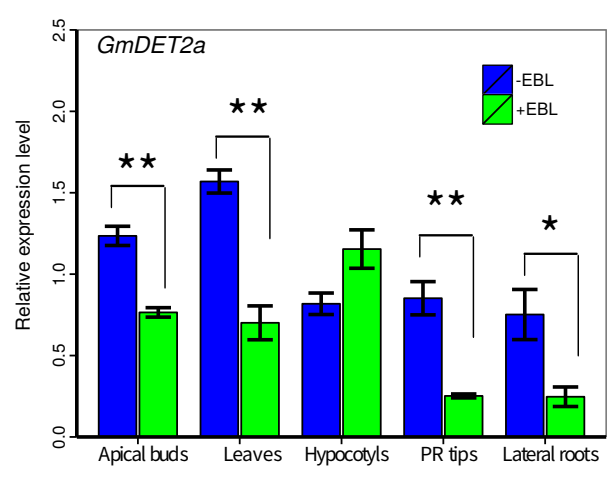

B

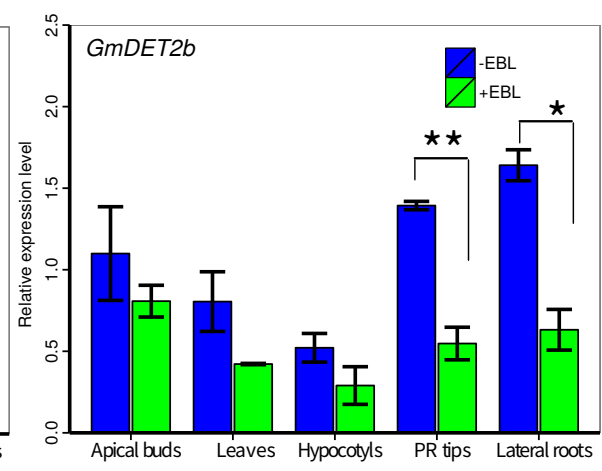

Figure 6. Responses of GmDET2a and GmDET2 $b$ to exogenous epibrassinosteroid (EBL) in soybean seedlings. RNA was extracted from five-day-old soybean YC03-3 treated with $10 \mu \mathrm{M}$ EBL for $6 \mathrm{~h}$ or not. Quantitative real-time PCR was exploited to detect the transcripts of GmDET2a (A) and GmDET2b (B) in apical buds, leaves, hypocotyls, primary root (PR) tips and lateral roots. Results are the means \pm SD from 4 replicates. Student's $t$-test was used to compare the differences between EBL treatment and control, respectively $\left({ }^{*}, 0.01<p<0.05 ;{ }^{* *}, 0.001<p<0.01\right)$. 


\subsection{Responses of GmDET2a and GmDET2b to Nutrient Deficiencies}

As our group is interested in plant nutrition, we also explored the responses of GmDET2a (Figure 7A) and GmDET2b (Figure 7B) to nutrient deficiencies. Soybean seedlings were starved of phosphate (-P), nitrogen (-N), potassium (-K) or sulfate (-S) for 14 days. Analysis by qRT-PCR showed that the transcripts of GmDET2 $a$ in leaves were decreased by $-\mathrm{S}$, and those of GmDET2b in leaves and roots were reduced by $-\mathrm{S}(p<0.05$; Figure $7 \mathrm{~B})$, while being upregulated by $-\mathrm{P}$ in both leaves and roots $(p<0.05)$. The transcripts of GmDET2a and GmDET2b were not regulated by $-\mathrm{N}$ or $-\mathrm{K}$ in either leaves or roots.

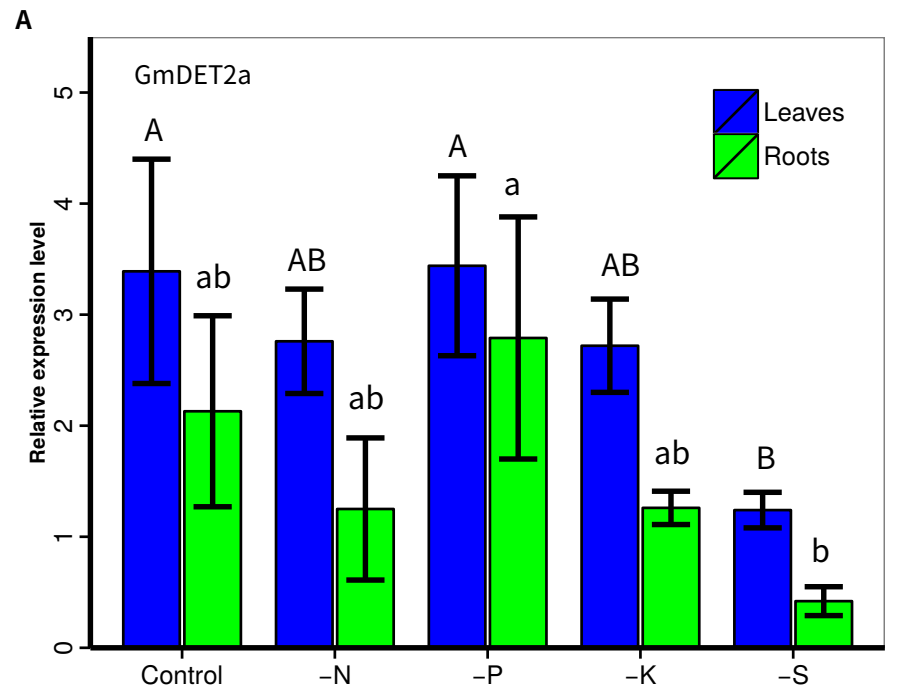

B

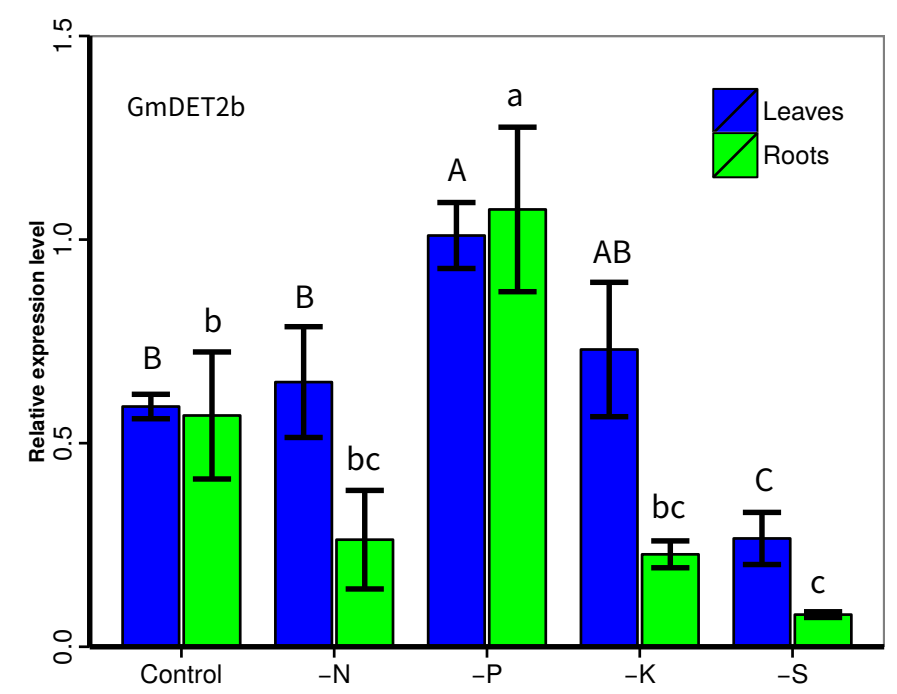

Figure 7. Responses of GmDET2a and DET2b to nutrient deficiency. Soybean YC03-3 was hydroponically cultured. After seven days, when the seedlings developed the first true leaf, soybean plants were transferred into a control nutrient solution, Pi-depleted (-P), nitrogen-depleted (-N), potassium-depleted (-K) and sulfate-depleted (-S) solution for 14 days. Quantitative real-time PCR was used to detect the expression levels of GmDET2a (A) and GmDET2b (B); GmEF1a was used as a reference gene. Results are the means \pm SD from four replicates. One-way ANOVA was employed to compare the difference, with uppercase letters or lowercase letters indicating the difference at the 0.05 level. L, leaves; R, roots. 


\subsection{Ectopic Expression of GmDET2a and GmDET2b in Atdet2-1 Rescued the Growth and Development Defects}

A loss of function mutation in DET2 affects the growth and development of Arabidopsis in both darkness and light $[5,35]$. To determine the functions of GmDET2 $a$ and GmDET2b, we constructed over-expression plasmids with the pCHF3 vector and transformed the loss-of-function mutant, Atdet2-1 [5]. We selected transgenic lines and identified single-copy lines based on the segregation ratio of 3:1. The transcript levels of GmDET2a and GmDET2b were explored in transgenic lines GmDET2aOX-20, GmDET2aOX-23, GmDET2bOX-16 and GmDET2bOX-23. As shown in Figure S1, the transgenic lines of GmDET2 $a$ and GmDET2b were overexpressing lines.

Consistent with previous results [5], the leaves of Atdet2-1 mutant are curled with a shorter petiole, as compared to wild-type (WT, Columbia (Col-0)) (Figure 8). As expected, when overexpressing GmDET2 $a$ or GmDET2b in Atdet2-1, the defective leaf growth and development were rescued. Images were taken after 15 days (Figure $8 \mathrm{~A}$ ) and 30 days of germination (Figure $8 \mathrm{~B}$ ).

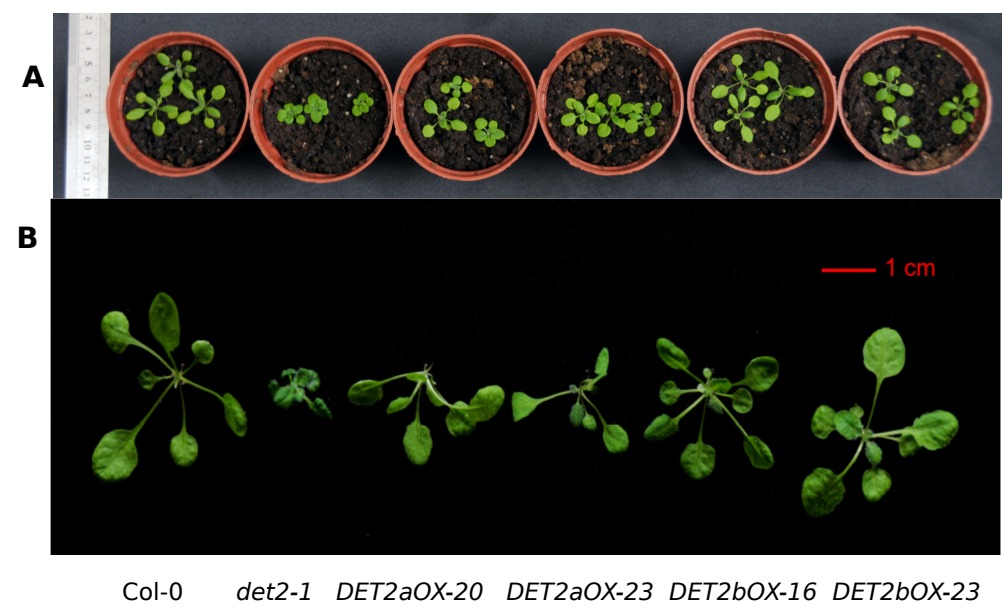

Figure 8. GmDET2a and GmDET2b rescued the leaf defects of Atdet2-1. Ectopic expression of GmDET2a or $G m D E T 2 b$ rescued the leaf defects in Atdet2-1 background. Arabidopsis seedlings were cultured in soils for 15 days (A) or 30 days (B), respectively. Scale bar, $1 \mathrm{~cm}$.

Consistent with previous study, the Atdet2-1 mutant is dwarf with short siliques (Figure 9A). We measured the height of transgenic Arabidopsis after 50 days of germination. The height of transgenic lines is higher in contrast to Atdet2-1 ( $p<0.05$, Figure 9B). This indicates that like AtDET2, GmDET2a or GmDET2B complement AtDET2, a steroid reductase.

BRs have been documented to play crucial roles in skotomorphogenesis [12], and the Atdet2-1 mutant has obviously different phenotypes from wild-type in the dark [5]. Hence, we compared the differences of Atdet2-1 and transgenic lines in the dark. In line with previous reports, different from Col-0, the Atdet2-1 mutant has a shorter hypocotyl $(0.86 \mathrm{~cm}$ VS $1.84 \mathrm{~cm})$ and primary roots and open cotyledons (Figure 10A). Overexpression of GmDET2a or GmDET2b in Atdet2-1 rescued the hypocotyl growth and primary root growth in the dark. The cotyledons in transgenic lines are less open in contrast to Atdet2-1. Data in Figure 10B verified that ectopic expression of GmDET2a or GmDET2b increases the hypocotyl growth in darkness. Compared to Atdet2-1, the hypocotyl length in the GmET2aOX-20, GmDET2aOX-23, GmDET2bOX-16 and GmDET2b-23 lines was increased by $110 \%, 87 \%$, $146 \%$ and $121 \%$, respectively $(p<0.05)$. Taken together, ectopic expression of GmDET2a and GmDET2b rescued the growth and development defects of Atdet2-1 in both the dark and light conditions. 


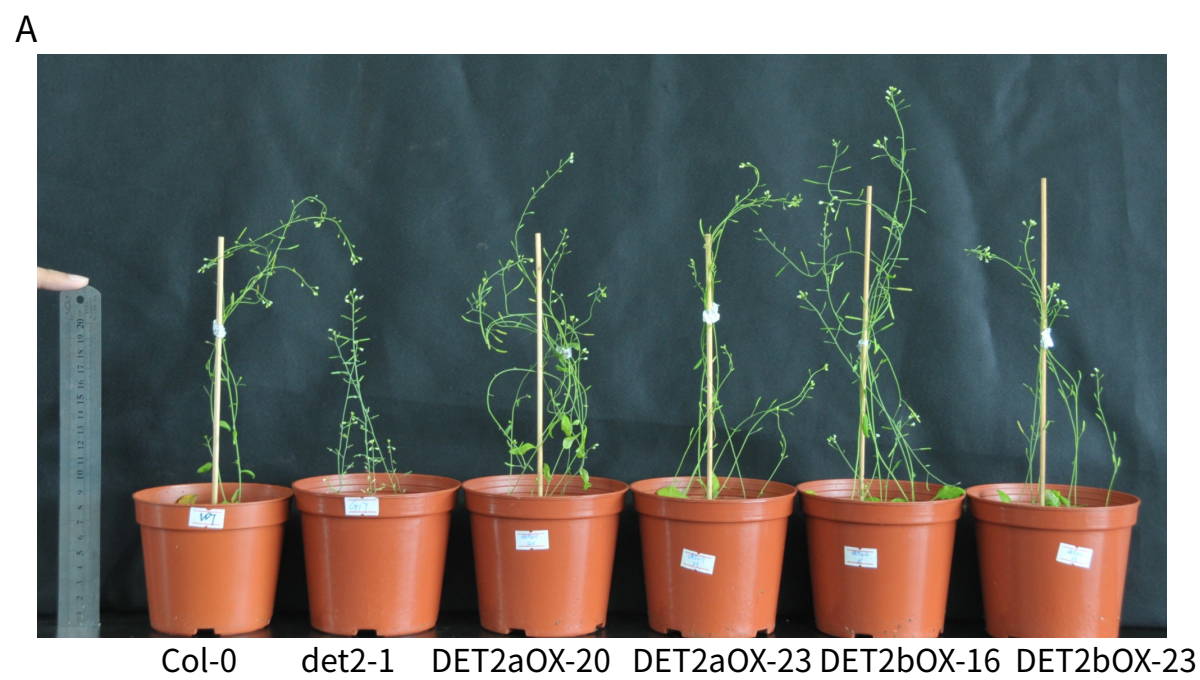

B
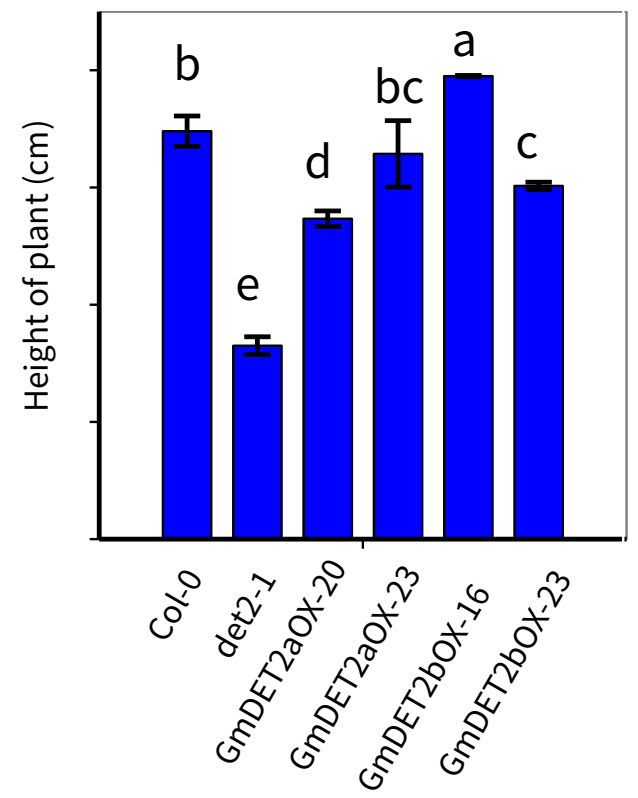

Figure 9. Ectopic expression of GmDET2a and GmDET2b rescued the height of Atdet2-1. The height of 50-day-old wild-type Col-0, Atdet2-1 and transgenic lines were measured (A). Results are the means \pm SD from four independent experiments, and one-way ANOVA was used to detect the differences among Col-0, Atdet2-1 and transgenic lines in the det2-1 background (B), with different letters indicating significant differences at the 0.05 level. 


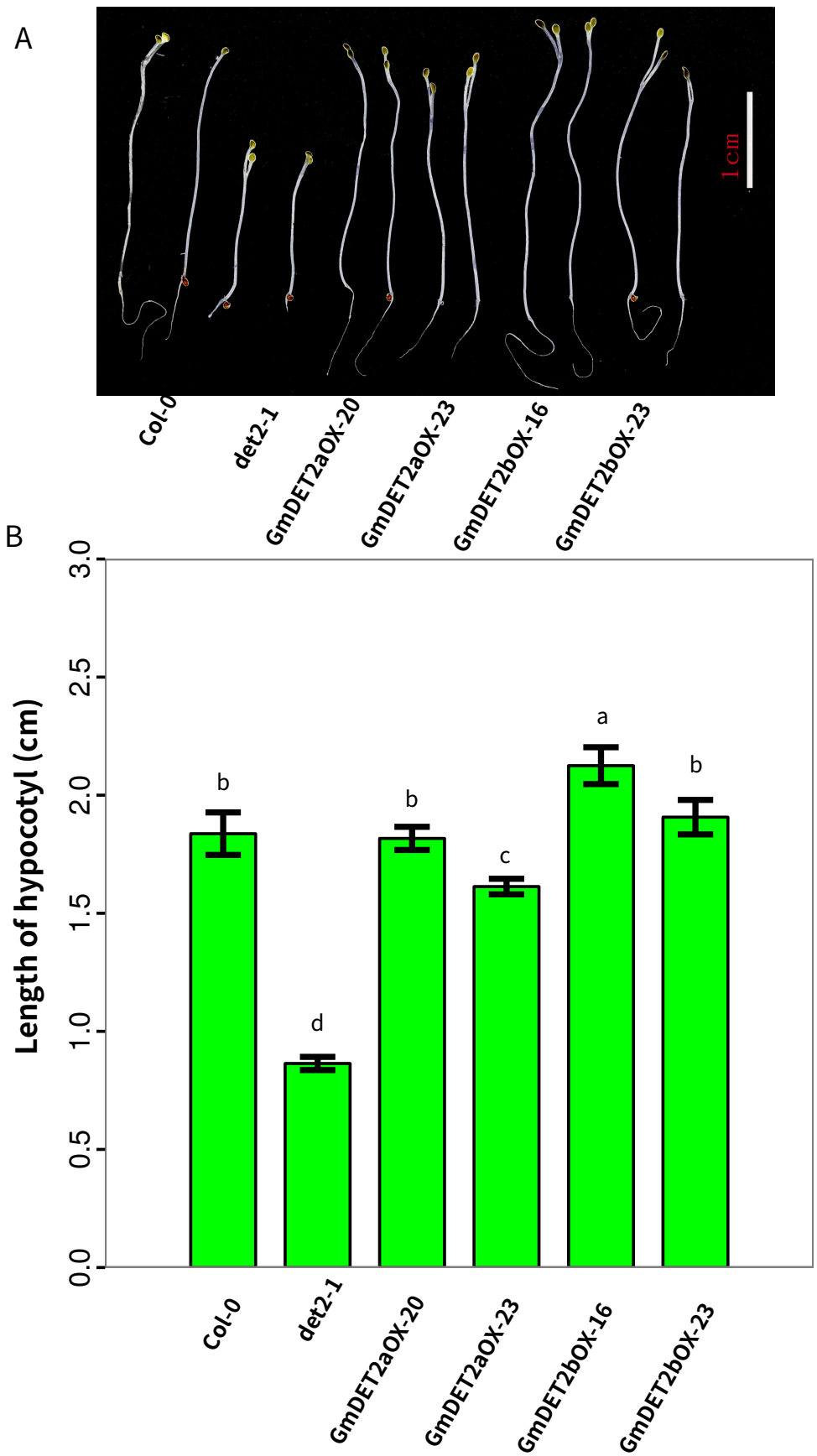

Figure 10. Ectopic expression of GmDET2a and GmDET2b rescued the skotomorphogenesis of Atdet2-1. Seeds of Col-0, Atdet2-1, transgenic lines were sown in half strength MS media and cultured in a growth chamber for one day with $16 \mathrm{~h}$ light $/ 8 \mathrm{~h}$ dark cycle to promote germination. Seeds were then cultured in the dark for five days and photographed (A). Scale bar, $1 \mathrm{~cm}$. The length of hypocotyls (B) was determined with ImageJ. Results are the means \pm SD from four replicates with 10 samples, respectively. Different letters indicate significant differences at the 0.05 level (one-way ANOVA).

2.7. Over-Expression of GmDET2a or GmDET2b Rescued the Responses of BR Biosynthesis-Related Genes to Exogenous BR

As reported, BR biosynthesis-related genes are feedback regulated by BR [47]. Therefore, we sought to determine whether the expression of GmDET2a or GmDET2b in the Atdet2-1 mutant background could rescue the responses of BR biosynthesis-related genes $C P D, D W F 4, B R 60 x-1$ and AtBR6ox-2. 
As demonstrated in Figure 11, compared to WT, the abundances of BR6ox-1, BR6ox-2, CPD and DWF4 were relative higher in the det2-1 mutant. When overexpressing GmDET2a in Atdet2-1, $B R 60 x-1, B R 60 x-2, C P D$ and DWF4 transcripts were significantly reduced $(p<0.05)$; with similar results observed when overexpresing GmDET2b in Atdet2-1. These results verified that soybean DET2a and DET2b complement the function of AtDET2 at the molecular level.

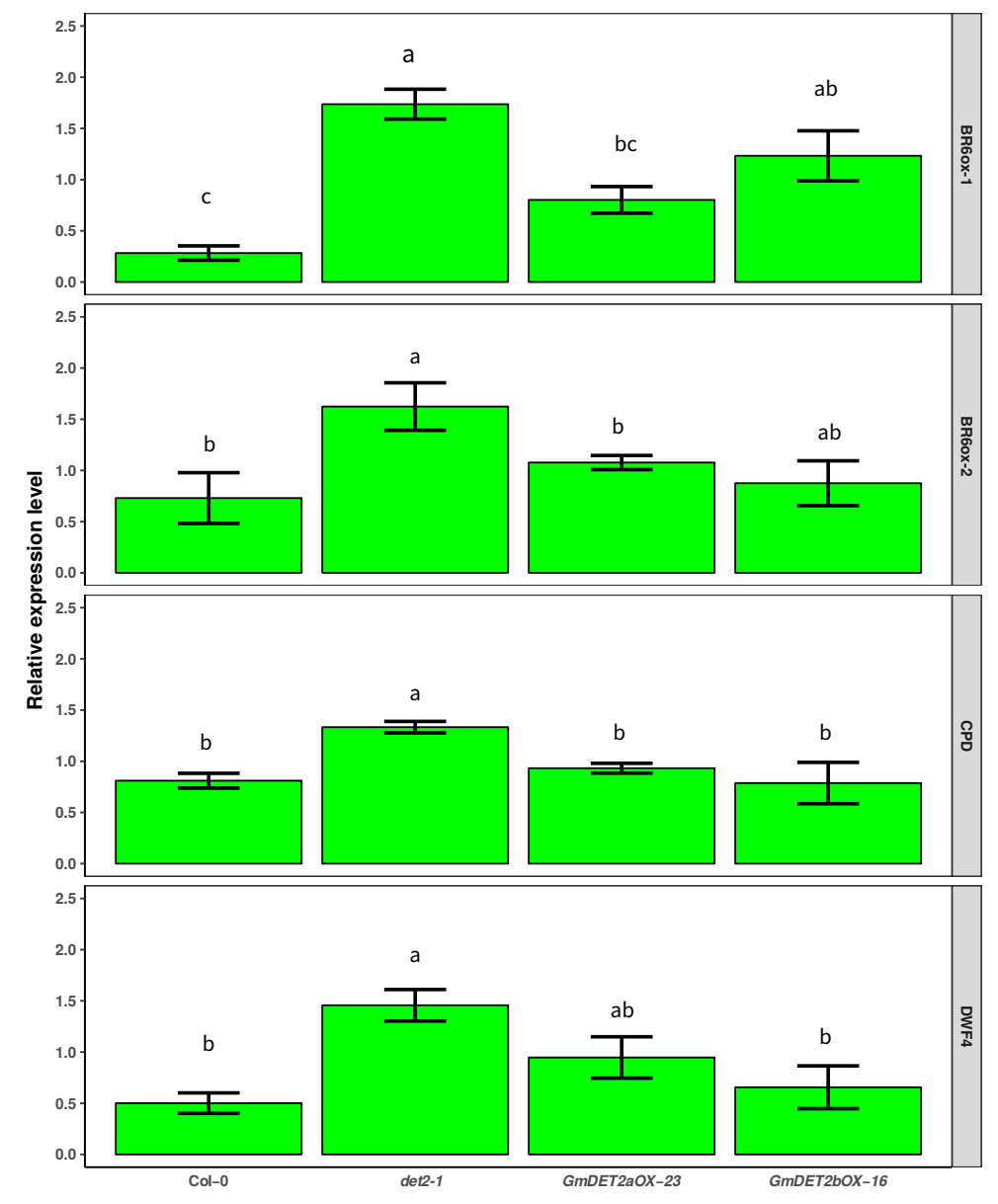

Figure 11. Effect of ectopic over-expression of GmDET2a and GmDET2b on the transcripts of BR biosynthesis-related genes in the Atdet 2-1 mutant. Arabidopsis seedlings were grown as described in the Materials and Methods. Quantitative RT-PCR was used to quantify the relative expression levels of BR6ox-1 (A), BR6ox-2 (B), CPD (C) and DWF4 (D) in det2-1 and transgenic lines. Results are the means \pm SD from four independent experiments with three technical repeats. One-way ANOVA was used to compare the difference among lines, with different letters indicating significant differences at the 0.05 level.

\section{Discussion}

Brassinosteroids are classic phytohormones, playing crucial roles in plant growth, development, abiotic and biotic stress responses [12]. Although the BR biosynthesis pathway in Arabidopsis has been extensively studied, the understanding of BR biosynthesis in soybean is poor. In this study, we identified eight soybean steroid reductase genes (GmDET2a to GmDET2h) and functionally characterized GmDET2a and GmDET2b at the physiological and molecular levels.

As early as 1990, BR was reported to stimulate adventitious rooting in soybean hypocotyl cuttings [37], and BR regulates nodulation and promotes drought tolerance [39,47]. The soybean genome encodes six BR receptors, and GmBRI1a and GmBRI1b have been reported [31,32]. Genes involved in soybean BR biosynthesis, unfortunately, are poorly understood. The BR biosynthetic 
process can be divided into two stages. The first stage sees the conversion of cycloartenol to campesterol, catalyzed by SMT1, FACKEL, DWF7/TE1, DWF5 and DWF1/DIM1 [47]. The second stage sees the conversion of campesterol to brassinosteroid, catalyzed by SAX1, DET2, DWF4, CPD, BR6ox-1 and BR6ox-2, respectively [47]. Arabidopsis DET2 has 80\% identity with animal steroid $5 \alpha$-dehydrogenase, including a highly conserved glutamate required for enzyme activity [35]. In Atdet2-1 and Atdet2-6 mutants, the conserved glutamate has been mutated into lysine, and Atdet $2-1$ has a $90 \%$ reduction in BR as compared to WT [35].

BR biosynthesis has been documented, thus far, in 61 plant species, including 53 angiosperm, 6 gymnosperms, 1 pteridophyte (Equisetum arvense) and one bryophyte (Marchantia polymorpha) [48]. Single-celled green freshwater algae (Chlorella vulgaris and Hydrodictyon reticulatum) and the marine brown alga Cystoseira myrica biosynthesize BRs, as well [49], indicating that BRs are highly conserved phytohormone. Based on studies in Arabidopsis and rice, DET2, encoding steroid reductase, a crucial enzyme in BR biosynthesis, catalyzes the rate-limiting step reaction [47]. Homologues of DET2 in tomato and cotton have been functionally identified, as well [50,51]

In this study, we found that the soybean genome encodes eight steroid reductase (Table 1). This indicates that brassinosteroid biosynthesis in soybean is more complex than in other species. GmDET2 $a$ and GmDET2 $b$ are intron-less genes, and consistent with AtDET2, GmDET2a and GmDET2b do not have the PF02544 steroid reductase signature (Table 1). Transgenic experiments verified that GmDET2 $a$ and GmDET2b function as steroid reductases in Arabidopsis, but more studies are needed to reveal the functions of the other six soybean DET genes. We also used PF02544 to search against the Phytozome database and retrieved 144 proteins containing steroid reductase/dehydrogenase domains from 19 species (Additional File 1). Evaluated by Prottest-2.4, JTT + G + F was selected as the best model to rebuild the evolutionary tree, utilizing MrBayes 3.2 software. As shown in Figure 3, steroid reductases were grouped into five subgroups. Except for Subgroup V, the other four subgroups contain steroid reductases from both Physcomitrella patens and Selaginella moellendorffii, implying that at least four ancestral steroid reductase genes exist in Plantae. Of note, fungus Ustilago maydis steroid $5 \alpha$-reductase complements Arabidopsis DET2 function [52], and overexpressing a human steroid $5 \alpha$-reductase rescues the growth and development of Atdet2-1 [35]. This evidence further verifies the existence of a common ancestor for steroid reductase between fungal, plant and mammalian proteins.

We found that in most branches, the Ka/Ks of steroid reductases was less than 1.0 (Figure 4), indicating that steroid reductases have been subjected to purifying selection. Ka/Ks values are also consistently less than one in BR receptor genes [32]. Similarly, as high as $76 \%$ analyzed jasmonate ZIM-domain (JAZ) genes from 13 monocot and dicot species have also been subject to purifying selection [53], and the $\mathrm{Ka} / \mathrm{Ks}$ of the squamosa promoter binding protein (SBP)-box genes in plants were less than 0.5 [54]. Our results support the notion that the $\mathrm{Ka} / \mathrm{Ks}$ of crucial genes in many plant species are less than 1.0 [55].

RNA-Seq data indicate that transcripts of eight soybean $D E T$ genes can be detected in roots, leaves, flowers, seeds and nodules (Figure 1B), implying that BR regulates the development and growth of these organs. Notably, the transcript abundances of GmDET2a and GmDET2b are higher in nodules and roots, indicating their important role in nodulation and rooting. The interaction between BR and auxin has been shown to stimulate lateral rooting [56], and BR signaling has been proven to boost root hair development [57].

Similarly, in tomato, the transcripts of LeDET2 can be detected in leaves, stems, roots, seeds and callus, with the highest abundance observed in leaves [50]. In this study, qRT-PCR results revealed that $G m D E T 2 a$ and $G m D E T 2 b$ have similar expression patterns (Figure 5) with higher expression in primary root tips, apical buds and lateral roots, implying that BR biosynthesis is active in these tissues. Differing from auxin, BR does not experience polar transport; thus, it is understandable that DET2 shows ubiquitous expression in primary roots, lateral roots, seeds and nodules. The future challenge will be to reveal the differing roles of soybean DET2s in different organs. 
We found that application of EBL for $6 \mathrm{~h}$ significantly decreased the transcripts of GmDET2a and $G m D E T 2 b$ in apical buds, leaves, PR tips and lateral roots (Figure 6A,B). DET2, CPD, DWF4, BR6ox-1 and $B R 60 x-2$ are key enzymes for BR biosynthesis [47] and are negatively regulated by exogenous $\mathrm{BR}$ or BR signal $[47,58]$. In line with this, we found that the transcripts of $C P D, D W F 4, B R 60 x-1$ and BR6ox-2 were downregulated in WT in contrast to Atdet2-1, but overexpressing GmDET2a or GmDET2b negatively regulated the levels of these four genes in Atdet2-1 (Figure 11). This is possibly due to the fact that ectopic expression of GmDET2a or GmDET2b in Atdet2-1 increases endogenous BR amounts. Molecular evidence also supports the idea that GmDET2a or GmDET2b encodes steroid reductase. Transcripts of DWF4 in WT are low, but those of DWF1, CPD and BRI1 are higher, indicating that transcription of DWF4 is downregulated by BR [47]. The fact that Arabidopsis CPD, DWF4 promoters contain binding elements of BZR1 or BES1 prompted us to conceive of the idea that the same cis-elements also exist in the promoter region of GmDET2a and GmDET2b. The homologue of BES1 or BZR1 can be blasted in soybean proteome.

Phosphate starvation induces the expression of GmDET2b, and $S$ starvation downregulates the transcripts of GmDET2a and GmDET2b (Figure 7). This hints at potential crosstalk between nutrients and BR biosynthesis. Whether it is phosphate- or sulfate-related transcription factors binding the promoters of GmDET2a or GmDET2b would be worthy of future study.

Atdet2-1 mutants exhibit a dwarf phenotype, and in darkness, the hypocotyl length of det2-1 is shorter than WT [5]. By ectopically expressing GmDET2a or GmDET2b in Atdet2-1, we show that GmDET2a and GmDET2b function as steroid reductase, as their overexpression rescued the defects of the mutant (curled leaf, reduced plant height in light and reduced hypocotyl length in dark) (Figures 8-10). In conclusion, we functionally identified GmDET2a and GmDET2b as steroid reductases involved in BR biosynthesis.

\section{Materials and Methods}

\subsection{Soybean and Arabidopsis Growth}

Glycine max genotype YC03-3 was used in this study. Soybean seeds were sterilized with $10 \%$ $\mathrm{NaClO}$ and germinated in sand until the cotyledon was open. The seedlings were then transferred to Hoagland solutions $(\mathrm{pH}=5.9)$ for three days, until the first true leaf developed. The seedlings were then cultured in $10 \mu \mathrm{M}$ EBL solution for $0 \mathrm{~h}$ and $6 \mathrm{~h}$, and the leaves were sprayed with $10 \mu \mathrm{M}$ EBL (+EBL) or $0 \mu \mathrm{M}$ EBL (-EBL). The apical buds, true leaves, hypocotyls, primary root tips and lateral roots were then removed and stored in liquid nitrogen. To study nutrient stress, germinating soybean seedlings were first cultured in complete Hoagland solution $(\mathrm{pH}=5.9)$ for seven days, as described [59], and the seedlings with the first fully-developed trifoliate leaves were transferred into phosphate (Pi)-sufficient (Control, $\left.250 \mu \mathrm{M} \mathrm{KH}{ }_{2} \mathrm{PO}_{4}\right)$ or phosphate deficient (-P) $\left(0 \mu \mathrm{M} \mathrm{KH}_{2} \mathrm{PO}_{4}\right)$ nutrient solution, for 14 days. For nitrogen starvation (-N), $\mathrm{NH}_{4} \mathrm{NO}_{3}$ was omitted, and $\mathrm{KCl}$ was substituted for $\mathrm{KNO}_{3}$. For potassium depletion (-K), $\mathrm{KNO}_{3}$ was omitted. For sulfate deficiency(-S), all $\mathrm{SO}_{4}^{2-}$ was substituted with $\mathrm{Cl}^{-1}$. All soybean solutions were aerated $15 \mathrm{~min}$ every $3 \mathrm{~h}$ and replaced with fresh solution every $48 \mathrm{~h}$. Soybean plants were grown under greenhouse conditions with 16-h light cycles.

Arabidopsis seeds were sterilized with 75\% ethanol for 2 min followed by $100 \%$ ethanol for $2 \mathrm{~min}$. Seeds were dried in a hood and sown on half strength MS media containing $1 \%$ sucrose and $0.8 \%$ agar (pH 5.8). Square Petri dishes were maintained in a growth chamber with a 16-h light $\left(100 \mu \mathrm{E} \mathrm{m}^{-2} \mathrm{~s}^{-1}\right) / 8$-h dark cycle and with a temperature cycle of $23^{\circ} \mathrm{C}$ light $/ 21^{\circ} \mathrm{C}$ dark. For the darkness treatment, the square Petri dishes were wrapped with aluminum foil. 


\subsection{Extraction of Genomic DNA, RNA and Reserve Transcription of $m R N A$}

Arabidopsis and soybean RNA were extracted with the TRIzol method. RNA were reverse transcribed through MLV-transcriptase.

\subsection{Determination of Soybean GmDET2 Family Structure}

Based on the full cDNA and genomic DNA sequences of GmDET2a to DET2h (www.phytozome.org), we exploited CSDS2.0 to plot the gene structure of GmDET2s.

\subsection{Alignment of DET2s}

T-coffee (http:/ / www.tcoffee.org/Projects/tcoffee/) was employed to align the sequence of DET2 from Glycine max, Arabidopsis, Solanum lycopersicum, Oryza sativa, Pisum sativum, Hordeum vulgare and Medicago truncatula.

\subsection{Phylogenetic Analysis}

Alignment of steroid reductases from Glycine max, Arabidopsis and Oryza sativa was done with ClustalW2.1. The evolutionary history was inferred by using the maximum likelihood method based on the Jones et al. w/freq.model [44]. The tree with the highest log likelihood (-2073.6839) is shown. The percentage of trees in which the associated taxa clustered together is shown next to the branches. Initial trees for the heuristic search were obtained automatically by applying neighbor-join and BioNJalgorithms to a matrix of pairwise distances estimated using a JTT model and then selecting the topology with the superior log likelihood value. A discrete gamma distribution was used to model evolutionary rate differences among sites $(5$ categories $(+G$, parameter $=5.1876)$ ). The tree is drawn to scale, with branch lengths measured in the number of substitutions per site. The analysis involved 20 amino acid sequences. All positions containing gaps and missing data were eliminated. There was a total of 74 positions in the final dataset. The bootstrap values were set to 1000 . Evolutionary analyses were conducted in MEGA6 [42].

A total of 105 steroid reductases from Physcomitrella patens, Selaginella moellendorffii, Cucumis sativus, Populus trichocarpa, Eucalyptus grandis, Citrus clementina, Solanum lycopersicum, Glycine max, Arabidopsis thaliana, Brachypodium distachyon, Oryza sativa, Amborella trichopoda, Gossypium raimondii, Aquilegia coerulea and Sorghum bicolor were selected to reconstruct the Bayesian phylogenies with MrBayes v3.2 [43]. In order to guarantee that the average standard deviation of split frequencies is below 0.05 , we set the runs of generation to be 100,000 . We employed a fixed Jones model (JTT) of amino acid substitution to reconstruct the phylogenetic tree (other parameters: nchain $=4$, samplefreq $=100$, samplefreq $=100$, printfreq $=100$ diagnfreq $=1000$ ).

\subsection{Analysis of Expression Patterns of Soybean DET2 Family at the Seedling Stage}

Based on the cDNA sequence and genomics sequences of GmDET2a and GmDET2b (www. phytozome.org), we designed specific primer pairs with PerlPrimer to detect their expression levels. Seven-day-old soybean cultivar YC03-3 seedlings were transferred to half strength Hoagland nutrient solutions to grow a further 7 days treated with different nutrient solutions. The roots, stems and leaves were sampled to extract total RNA. Quantitative real-time PCR was used to test the expression levels of GmDET2 $a$ and GmDET2b, and soybean EF1a was used to normalize the data. Forty qRT-PCR cycles were run $\left(95^{\circ} \mathrm{C} 1 \mathrm{~min}, 95^{\circ} \mathrm{C} 15 \mathrm{~s}, 60^{\circ} \mathrm{C} 15 \mathrm{~s}, 72{ }^{\circ} \mathrm{C} 30 \mathrm{~s}, 72{ }^{\circ} \mathrm{C} 45 \mathrm{~s}\right)$, and Rotorgen software was used to calculate the PCR results. Data were from three independent biological experiments. 


\subsection{Overexpression of GmDET2a and GmDET2b in Arabidopsis det2}

As the coding region sequences of GmDET2a and GmDET2b are nearly $100 \%$ identical, we did two rounds of PCR to clone the open reading frame of GmDET2a and GmDET2b. First, we designed PCR primers in the 5 UTR and 3 UTR of GmDET2 $a$ and GmDET2b and then used the common forward and reverse primers GmDET2a/b.oxF and GmDET2a/b.oxR that contain the KpnI and SalI restriction sites, respectively, to amplify the coding fragment. The coding fragments of GmDET2a and GmDET2b and pCHF3 plasmid were digested with KpnI and SalI, respectively, and the digested pCHF3, GmDET2a and GmDET2b coding fragments were ligated using DNA ligase. The Atdet2-1 mutant was transformed with pCHF3-GmDET2a or pCHF3-GmDET2b using the floral dip method and the GV3101 agrobacterium strain [60]. Transformed lines were selected in media containing $50 \mu \mathrm{g} / \mathrm{mL}$ kanamycin. The single copy T-DNA transformed lines were determined based the 3:1 segregation ratio. The homozygous F3 were used in further experiments.

\subsection{Phenotypic Analysis of Transgenic Arabidopsis}

Seeds of wild-type Col-0, Atdet2-1 and overexpression lines in mutant background, as indicated, were stratified in $4{ }^{\circ} \mathrm{C}$ for 2 days to break dormancy and then sown into soils, and after being cultured as indicated, seedling height was measured with a ruler.

\subsection{Determination of the Expression Levels of BR Biosynthesis-Related Genes in Col-0, det2-1 and Transgenic Lines}

To test the expression levels of BR biosynthesis-related genes CPD, DWF4, BR6ox-1 and BR6ox-2, 3-week-old Arabidopsis seedlings of Col-0, Atdet2-1 and overexpression lines in mutant background were transplanted to the soil for one month under standard growth conditions, and then, the whole plants were used to extract total RNA with the TRIzol method. cDNAs were obtained with reverse transcriptase-mediated reactions. Transcript abundance of CPD, DWF4, BR6ox-1 and BR6ox-2 was determined using qRT-PCR, and AtEF1a was used as a reference gene to normalize the qRT-PCR results. Specific primer pairs are listed in Supplementary Table S2.

\subsection{Data Analysis}

All data were analyzed with Excel2003. Student's $t$-test and one-way ANOVA were employed to compare the differences. R 3.0.1 package [61], ggplot2 and gplots 2.12.1 [62] were used to draw figures and the heat map, respectively.

\section{Conclusions}

The soybean genome has eight genes that encode steroid reductase (GmDET2a to GmDET2h), and GmDET2a and GmDET2b were negatively regulated by exogenous BR. BR reductases in plants were subjected to purifying selection during evolution. Ectopic overexpression of GmDET2a and GmDET2b in Atdet2-1 rescues the BR biosynthesis deficiency-related growth and development defects of the det2-1 under dark and light conditions.

Supplementary Materials: The following are available online at www.mdpi.com/1422-0067/19/3/726/s1.

Acknowledgments: This study was partially supported by NSFC (No. 31572184). We thank Joanne Chory for providing det2-1 seeds and Jennifer Mach for her comments and help in English writing.

Author Contributions: Weige Huo participated in the study design, carried out the experiments and data analysis and drafted the manuscript. Bodi Li, Jiebing Kuang, Pingan He and Zhihao Xu carried out the experiments and analyzed the data. Jinxiang Wang conceived and designed experiments, analyzed the data and authored the manuscript. All authors have read and approved the final manuscript.

Conflicts of Interest: The authors declare no conflict of interest. 


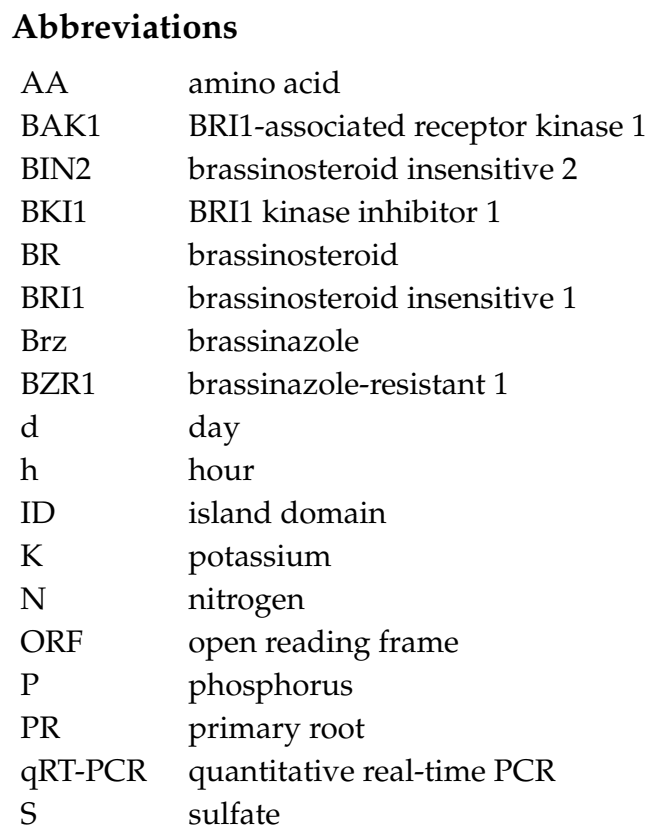

\section{References}

1. Müssig, C.; Shin, G.H.; Altmann, T. Brassinosteroids promote root growth in Arabidopsis. Plant Physiol. 2003, 133, 1261-1271.

2. Hacham, Y.; Holland, N.; Butterfield, C.; Ubeda-Tomas, S.; Bennett, M.J.; Chory, J.; Savaldi-Goldstein, S. Brassinosteroid perception in the epidermis controls root meristem size. Development 2011, 138, 839-848.

3. Kim, B.H.; Kim, S.Y.; Nam, K.H. Genes encoding plant-specific class III peroxidases are responsible for increased cold tolerance of the brassinosteroid-insensitive 1 mutant. Mol. Cells 2012, 34, 539-548.

4. Leubner-Metzger, G. Brassinosteroids and gibberellins promote tobacco seed germination by distinct pathways. Planta 2001, 213, 758-763.

5. Chory, J.; Nagpal, P.; Peto, C.A. Phenotypic and Genetic Analysis of det2, a New Mutant That Affects Light-Regulated Seedling Development in Arabidopsis. Plant cell 1991, 3, 445-459.

6. Szekeres, M.; Németh, K.; Koncz-Kálmán, Z.; Mathur, J.; Kauschmann, A.; Altmann, T.; Rédei, G.P.; Nagy, F.; Schell, J.; Koncz, C. Brassinosteroids rescue the deficiency of CYP90, a cytochrome P450, controlling cell elongation and de-etiolation in Arabidopsis. Cell 1996, 85, 171-182.

7. Bai, M.Y.; Fan, M.; Oh, E.; Wang, Z.Y. A triple helix-loop-helix/basic helix-loop-helix cascade controls cell elongation downstream of multiple hormonal and environmental signaling pathways in Arabidopsis. Plant Cell 2012, 24, 4917-4929.

8. Ferguson, B.J.; Ross, J.J.; Reid, J.B. Nodulation phenotypes of gibberellin and brassinosteroid mutants of pea. Plant Physiol. 2005, 138, 2396-2405.

9. Nakashita, H.; Yasuda, M.; Nitta, T.; Asami, T.; Fujioka, S.; Arai, Y.; Sekimata, K.; Takatsuto, S.; Yamaguchi, I.; Yoshida, S. Brassinosteroid functions in a broad range of disease resistance in tobacco and rice. Plant J. 2003, 33, 887-898.

10. Dhaubhadel, S.; Browning, K.S.; Gallie, D.R.; Krishna, P. Brassinosteroid functions to protect the translational machinery and heat-shock protein synthesis following thermal stress. Plant J. 2002, 29, 681-691.

11. Bajguz, A.; Hayat, S. Effects of brassinosteroids on the plant responses to environmental stresses. Plant Physiol. Biochem. 2009, 47, 1-8.

12. Li, J.; Chory, J. A putative leucine-rich repeat receptor kinase involved in brassinosteroid signal transduction. Cell 1997, 90, 929-938.

13. Wang, Z.Y.; Nakano, T.; Gendron, J.; He, J.; Chen, M.; Vafeados, D.; Yang, Y.; Fujioka, S.; Yoshida, S.; Asami, T.; et al. Nuclear-localized BZR1 mediates brassinosteroid-induced growth and feedback suppression of brassinosteroid biosynthesis. Dev. Cell 2002, 2, 505-513. 
14. Wang, X.; Chory, J. Brassinosteroids regulate dissociation of BKI1, a negative regulator of BRI1 signaling, from the plasma membrane. Science 2006, 313, 1118-1122.

15. Li, J.; Wen, J.; Lease, K.A.; Doke, J.T.; Tax, F.E.; Walker, J.C. BAK1, an Arabidopsis LRR receptor-like protein kinase, interacts with BRI1 and modulates brassinosteroid signaling. Cell 2002, 110, 213-222.

16. Li, J.; Nam, K.H.; Vafeados, D.; Chory, J. BIN2, a new brassinosteroid-insensitive locus in Arabidopsis. Plant Physiol. 2001, 127, 14-22.

17. Li, J.; Nam, K.H. Regulation of brassinosteroid signaling by a GSK3/SHAGGY-like kinase. Science 2002, $295,1299-1301$.

18. He, J.X.; Gendron, J.M.; Yang, Y.; Li, J.; Wang, Z.Y. The GSK3-like kinase BIN2 phosphorylates and destabilizes BZR1, a positive regulator of the brassinosteroid signaling pathway in Arabidopsis. Proc. Natl. Acad. Sci. USA 2002, 99, 10185-10190.

19. Zhao, J.; Peng, P.; Schmitz, R.J.; Decker, A.D.; Tax, F.E.; Li, J. Two putative BIN2 substrates are nuclear components of brassinosteroid signaling. Plant Physiol. 2002, 130, 1221-1229.

20. Tang, W.; Yuan, M.; Wang, R.; Yang, Y.; Wang, C.; Oses-Prieto, J.A.; Kim, T.W.; Zhou, H.W.; Deng, Z.; Gampala, S.S.; et al. PP2A activates brassinosteroid-responsive gene expression and plant growth by dephosphorylating BZR1. Nat. Cell Biol. 2011, 13, 124-131.

21. Yin, Y.; Vafeados, D.; Tao, Y.; Yoshida, S.; Asami, T.; Chory, J. A new class of transcription factors mediates brassinosteroidregulated gene expression in Arabidopsis. Cell 2005, 120, 249-259.

22. Peng, P.; Yan, Z.; Zhu, Y.; Li, J. Regulation of the Arabidopsis GSK3-like kinase BRASSINOSTEROIDINSENSITIVE 2 through proteasome-mediated protein degradation. Mol. Plant 2008, 1, 338-346.

23. Cano-Delgado, A.; Yin, Y.; Yu, C.; Vafeados, D.; Mora-Garca, S.; Cheng, J.C.; Nam, K.H.; Li, J.; Chory, J. BRL1 and BRL3 are novel brassinosteroid receptors that function in vascular differentiation in Arabidopsis. Development 2004, 131, 5341-5351.

24. Zhou, A.; Wang, H.; Walker, J.C.; Li, J. BRL1, a leucine-rich repeat receptor-like protein kinase, is functionally redundant with BRI1 in regulating Arabidopsis brassinosteroid signaling. Plant J. 2004, 40, 399-409.

25. Yamamuro, C.; Ihara, Y.; Wu, X.; Noguchi, T.; Fujioka, S.; Takatsuto, S.; Ashikari, M.; Kitano, H.; Matsuoka, M. Loss of function of a rice brassinosteroid insensitive1 homolog prevents internode elongation and bending of the lamina joint. Plant Cell 2000, 12, 1591-1606.

26. Nakamura, A.; Fujioka, S.; Sunohara, H.; Kamiya, N.; Hong, Z.; Inukai, Y.; Miura, K.; Takatsuto, S.; Yoshida, S.; Ueguchi-Tanaka, M.; et al. The role of OsBRI1 and its homologous genes, OsBRL1 and OsBRL3, in rice. Plant Physiol. 2006, 140, 580-590.

27. Montoya, T.; Nomura, T.; Farrar, K.; Kaneta, T.; Yokota, T.; Bishop, G.J. Cloning the tomato curl3 gene highlights the putative dual role of the leucine-rich repeat receptor kinase tBRI1/SR160 in plant steroid hormone and peptide hormone signaling. Plant cell 2002, 14, 3163-3176.

28. Nomura, T.; Bishop, G.J.; Kaneta, T.; Reid, J.B.; Chory, J.; Yokota, T. The LKA gene is a brassinosteroid insensitive 1 homolog of pea. Plant J. 2003, 36, 291-300.

29. Chono, M.; Honda, I.; Zeniya, H.; Yoneyama, K.; Saisho, D.; Takeda, K.; Takatsuto, S.; Hoshino, T.; Watanabe, Y. A semidwarf phenotype of barley uzu results from a nucleotide substitution in the gene encoding a putative brassinosteroid receptor. Plant Physiol. 2003, 133, 1209-1219.

30. Sun, Y.; Fokar, M.; Asami, T.; Yoshida, S.; Allen, R.D. Characterization of the brassinosteroid insensitive 1 genes of cotton. Plant Mol. Biol. 2004, 54, 221-232.

31. Wang, M.; Sun, S.; Wu, C.; Han, T.; Wang, Q. Isolation and characterization of the brassinosteroid receptor gene (GmBRI1) from Glycine max. Int. J. Mol. Sci. 2014, 15, 3871-3888.

32. Peng, S.; Tao, P.; Xu, F.; Wu, A.; Huo, W.; Wang, J. Functional Characterization of Soybean Glyma04g39610 as a Brassinosteroid Receptor Gene and Evolutionary Analysis of Soybean Brassinosteroid Receptors. Int. J. Mol. Sci. 2016, 17, 897.

33. Bajguz A, T.A. The chemical characteristic and distribution of brassinosteroids in plants. Phytochemistry 2003, 62, 1027-1046.

34. Fujioka, S.; Yokota, T. Biosynthesis and metabolism of brassinosteroids. Annu. Rev. Plant Biol. 2003, 54, 137-164.

35. Li, J.; Biswas, M.; Chao, A.; Russell, D.; Chory, J. Conservation of function between mammalian and plant steroid 5 $\alpha$-reductases. Proc. Natl. Acad. Sci. USA 1997, 94, 3554-3559. 
36. Noguchi, T.; Fujioka, S.; Choe, S.; Takatsuto, S.; Yoshida, S.; Yuan, H.; Feldmann, K.; Tax, F. Brassinosteroidinsensitive dwarf mutants of Arabidopsis accumulate brassinosteroids. Plant Physiol. 1999, 121, 743-752.

37. Sathiyamoorthy, P.; Nakamura, S. In vitro root induction by 24-epibrassinolide on hypocotyl segments of soybean [Glycine max (L.) Merr.]. Plant Growth Regul. 1990, 9, 73-76.

38. Zurek, D.M.; Rayle, D.L.; McMorris, T.C.; Clouse, S.D. Investigation of Gene Expression, Growth Kinetics, and Wall Extensibility during Brassinosteroid-Regulated Stem Elongation. Plant Physiol. 1994, 104, 505-513.

39. Zhang, M.; Zhai, Z.; Tian, X.; Duan, L.; Li, Z. Brassinolide alleviated the adverse effect of water deficits on photosynthesis and the antioxidant of soybean (Glycine max L.). Plant Growth Regul. 2008, 56, 257-264.

40. Li, J.; Nagpal, P.; Vitart, V.; McMorris, T.; Chory, J. A role for brassinosteroids in light-dependent development of Arabidopsis. Science 1996, 272, 398-401.

41. Severin, A.J.; Woody, J.L.; Bolon, Y.T.; Joseph, B.; Diers, B.W.; Farmer, A.D.; Muehlbauer, G.J.; Nelson, R.T.; Grant, D.; Specht, J.E.; et al. RNA-Seq Atlas of Glycine max: A guide to the soybean transcriptome. BMC Plant Biol. 2010, 10, 160.

42. Tamura, K.; Stecher, G.; Peterson, D.; Filipski, A.; Kumar, S. MEGA6: Molecular evolutionary genetics analysis version 6.0. Mol. Biol. Evol. 2013, 30, 2725-2729.

43. Huelsenbeck, J.P.; Ronquist, F. MRBAYES: Bayesian inference of phylogenetic trees. Bioinformatics 2001, 17, 754-755.

44. Jones, D.; Taylor, W.; Thornton, J. The rapid generation of mutation data matrices from protein sequences. Comput. Appl. Biosci. 1992, 8, 275-282.

45. Bancos, S.; Nomura, T.; Sato, T.; Moln'ar, G.; Bishop, G.J.; Koncz, C.; Yokota, T.; Nagy, F.; Szekeres, M. Regulation of transcript levels of the Arabidopsis cytochrome p450 genes involved in brassinosteroid biosynthesis. Plant Physiol. 2002, 130, 504-513.

46. Tanaka, K.; Asami, T.; Yoshida, S.; Nakamura, Y.; Matsuo, T.; Okamoto, S. Brassinosteroid homeostasis in Arabidopsis is ensured by feedback expressions of multiple genes involved in its metabolism. Plant Physiol. 2005, 138, 1117-1125.

47. Terakado, J.; Fujihara, S.; Goto, S.; Kuratani, R.; Suzuki, Y.; Yoshida, S.; Yoneyama, T. Systemic Effect of a Brassinosteroid on Root Nodule Formation in Soybean as Revealed by the Application of Brassinolide and Brassinazole. Soil Sci. Plant Nutr. 2005, 51, 389-395.

48. Kutschera, U.; Wang, Z.Y. Brassinosteroid action in flowering plants: A Darwinian perspective. J. Exp. Bot. 2012, 63, 3511-3522.

49. Hayat, S.; Ahmad, A. Brassinosteroids: A Class of Plant Hormone; Springer: New York, NY, USA, 2011.

50. Rosati, F.; Bardazzi, I.; De Blasi, P.; Simi, L.; Scarpi, D.; Guarna, A.; Serio, M.; Racchi, M.L.; Danza, G. 5alpha-Reductase activity in Lycopersicon esculentum: cloning and functional characterization of LeDET2 and evidence of the prealsence of two isoenzymes. J. Steroid Biochem. Mol. Biol. 2005, 96, 287-299.

51. Luo, M.; Xiao, Y.; Li, X.; Lu, X.; Deng, W.; Li, D.; Hou, L.; Hu, M.; Li, Y.; Pei, Y. GhDET2, a steroid $5 \alpha$-reductase, plays an important role in cotton fiber cell initiation and elongation. Plant J. 2007, 51, 419-430.

52. Basse, C.W.; Kerschbamer, C.; Brustmann, M.; Altmann, T.; Kahmann, R. Evidence for a Ustilago maydis steroid 5alpha-reductase by functional expression in Arabidopsis det2-1 mutants. Plant Physiol. 2002, 129, 717-732.

53. Singh, A.P.; Pandey, B.K.; Deveshwar, P.; Narnoliya, L.; Parida, S.K.; Giri, J. JAZ Repressors: Potential Involvement in Nutrients Deficiency Response in Rice and Chickpea. Front. Plant Sci. 2015, 6, 975.

54. Zhang, S.D.; Ling, L.Z.; Yi, T.S. Evolution and divergence of SBP-box genes in land plants. BMC Genom. 2015, 16, 787.

55. Victoria, F.d.C.; Bervald, C.M.P.; da Maia, L.C.; de Sousa, R.O.; Panaud, O.; de Oliveira, A.C. Phylogenetic relationships and selective pressure on gene families related to iron homeostasis in land plants. Genome 2012, 55, 883-900.

56. Bao, F.; Shen, J.; Brady, S.R.; Muday, G.K.; Asami, T.; Yang, Z. Brassinosteroids interact with auxin to promote lateral root development in Arabidopsis. Plant Physiol. 2004, 134, 1624-1631.

57. Cheng, Y.; Zhu, W.; Chen, Y.; Ito, S.; Asami, T.; Wang, X. Brassinosteroids control root epidermal cell fate via direct regulation of a MYB-bHLH-WD40 complex by GSK3-like kinases. eLife 2014, 3, e02525.

58. He, J.X.; Gendron, J.M.; Sun, Y.; Gampala, S.S.L.; Gendron, N.; Sun, C.Q.; Wang, Z.Y. BZR1 is a transcriptional repressor with dual roles in brassinosteroid homeostasis and growth responses. Science 2005, 307, 1634-1638. 
59. Xu, F.; Liu, Q.; Chen, L.; Kuang, J.; Walk, T.; Wang, J.; Liao, H. Genome-wide identification of soybean microRNAs and their targets reveals their organ-specificity and responses to phosphate starvation. BMC Genom. 2013, 14, 66.

60. Clough, S.J.; Bent, A.F. Floral dip: A simplified method forAgrobacterium-mediated transformation of Arabidopsis thaliana. Plant J. 1998, 16, 735-743.

61. R Core Team. R: A Language and Environment for Statistical Computing; R Foundation for Statistical Computing: Vienna, Austria, 2013.

62. Warnes, G.R.; Ben, B.; Lodewijk, B.; Robert, G.; Wolfgang, H.; Andy, L.; Thomas, L. GR Gplots: Various R programming tools for plotting data. R Packag. Version 2009, 3, 1.

(C) 2018 by the authors. Licensee MDPI, Basel, Switzerland. This article is an open access article distributed under the terms and conditions of the Creative Commons Attribution (CC BY) license (http://creativecommons.org/licenses/by/4.0/). 